\section{Problematisch: Die Do-it-yourself-Patchtests durch Zahnärzte}

\section{Eine Sensibilisierung auf Zahnersatzmaterialien läßt sich im Einzelfall mit dem Epikutantest nur schwer beweisen. Experten warnen vor einer unkritischen Anwendung und Beurteilung des Tests, wie dies zunehmend in Zahnartzpraxen zu beobachten ist. Hier kann es z.B. durch den undurchdachten Einsatz selbstgestrickter Epikutantests auf Kunststoffe zu einer weiteren Zunahme von Sensibilisierungen kommen.}

D ie Allergologie ist in Zahnarztpraxen nicht besonders gut aufgehoben. Gerade im Dentalbereich, gebe es nach den Worten von G. Richter, Klinik und Poliklinik für Dermatologie der TU Dresden, viele Fallstricke. So sei die Unterscheidung einer allergischen von einer irritativen Reaktion bei vielen Dentallegierungen nur schwer möglich. Eine große Multizenterstudie habe gezeigt, daß der Reaktionsindex nur bei Nickeldentallegierungen positiv war (beweisend für eine allergische Reaktion), während er bei Kobaltlegierungen um 0 lag und bei Titan und Goldlegierungen sogar nega-

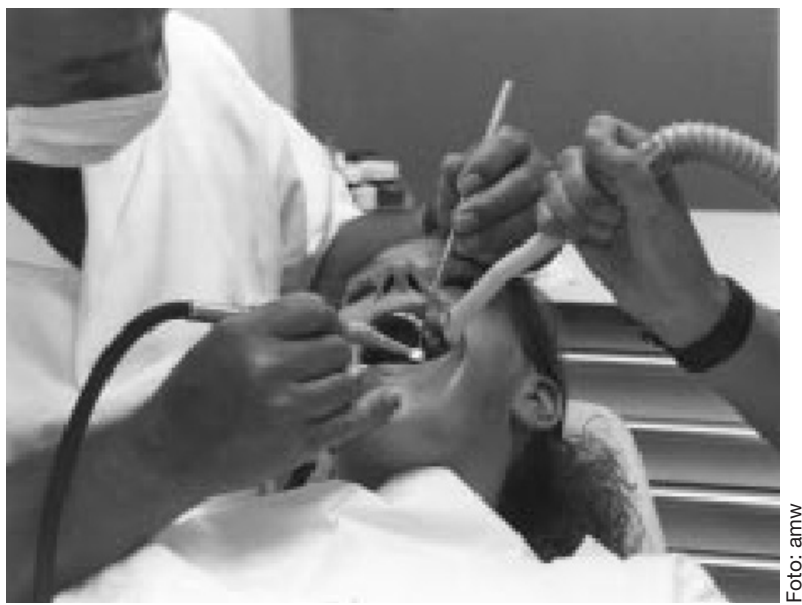

Längst nicht mehr nur die Angst vorm Bohren macht den Besuch beim Dentisten unerfreulich. Nun kommt auch noch die Furcht vor Irritationen durch ,allergenes“ Dentalmaterial hinzu. tiv ausfiel.

Die Möglichkeit, eine spätere allergische Reaktion bei den Patienten durch einen Epikutantest vorherzusagen, schätzte Richter deshalb als gering ein. Allenfalls sei dies möglich, wenn alle Inhaltsstoffe einzeln in mehrfachen Tests untersucht würden.

Bei einer nachgewiesenen Sensibilisierung auf Goldsalze stelle sich zudem die Frage der klinischen Relevanz. Denn die Mundschleimhaut reagiere wesentlich unempfindlicher auf allergische Reaktionen als die Haut.

Schließlich gebe es auch die Möglichkeit, den Patienten durch eine ko. Ein Hersteller von kommerziellen Dentalacrylat-Testsubstanzen habe nach einer Reihe von Sensibilisierungen die Konzentration der Allergene in dem Testansatz drastisch senken müssen.
Als geradezu fahrlässig bezeichnete Richter vor diesem Hintergrund die zunehmende Praxis von Zahnärzten, welche die verwendeten Kunststoffe in einem selbstgestrickten Epikutantest vorher auf der Haut der Patienten testen. Diese dürften sich dann nicht wundern, wenn die Zahl der sensibilisierten Patienten ständig zunehme, warnte Richter.

(rme)

Quelle: Vortrag von R. Richter (Dresden) anläßlich de 25. Kongresses des Ärzteverbandes Deutscher Allergologen, Göttingen 1998.

\section{Der „Mutter-und- Kind"-Pricktest}

Der Pricktest kann bei vermuteten Nahrungsmittelallergien (NMA) helfen, die Spreu vom Weizen zu trennen. Nach Angaben von K. Brockow wird er an der TU München bereits bei Kleinkindern durchgeführt. Um einen Schreianfall des Kindes zu vermeiden, werde das Kind von der Mutter (oder dem Vater) auf der Trage liegend gehalten, während der Test in aller Schnelle durchgeführt werde. Brockow: „Wenn Sie geschickt sind, haben Sie den Test durchgeführt, bevor das Kind etwas merkt."

Erfahrungen am UKE in Hamburg hätten gezeigt, daß Kinder auf Hühnereiweiß mit einem Anteil von $35 \%$ noch häufiger positiv reagieren als auf Kuhmilch (23\%). Sensibilisierungen auf Weizen $(8 \%)$ und Soja $(9 \%)$ sind dagegen seltener.

Der Pricktest hat nach Angaben Brockows einen negativen prädiktiven Wert von 98\%. Seine Schwachstelle ist der positive prädiktive Wert von nur $65 \%$. Bei einem positiven Ergebnis müsse deshalb die Diagnostik weitergeführt werden. Oft wird dann ein doppelblinder Provokationstest (DBPCFC), der Goldstandard der NMADiagnostik, notwendig.

(rme) 